\title{
LOS NIÑOS ANTE LA PÉRDIDA DE UNO DE LOS PROGENITORES: REVISIÓN DE PAUTAS DE COMUNICACIÓN EFICACES
}

\section{CHILDREN AT THE LOSS OF A PARENT: REVIEW OF EFFECTIVE COMMUNICATION STANDARDS}

Victoria Mesquida Hernando, Raquel Seijas Gómez y Mónica Rodríguez Enríquez

Hospital Universitario Son Espases. Palma de Mallorca.

Resumen

Objetivo: La muerte es un tema tabú en la sociedad actual, y por ello es común que los padres tengan dudas acerca de cómo abordar este tema con los menores. Los objetivos de este trabajo son: 1) describir el concepto evolutivo de muerte y la forma de afrontamiento de niños y adolescentes ante una pérdida y 2) hacer una revisión bibliográfica de las principales recomendaciones y pautas para abordar el proceso de comunicación y preparación de los niños ante esta situación.

Método: Se ha hecho una búsqueda bibliográfica sistemática en bases de datos primarias y secundarias (TripDatbase, PsycInfo, PubMed, Cochrane Library, Psicodoc) combinando diferentes términos clave.

Resultados: La mayor parte del material revisado procede del ámbito anglosajón. En nuestro país la mayoría tiene un formato divulgativo, y no consta a los autores de este trabajo la publicación de investigaciones regladas. Sin embargo sí es posible extraer de todo el material revisado una serie de pautas generales que facilitan la comunicación con los menores en esas situaciones.

Conclusiones: La comunicación adecuada y en base a unas pautas establecidas contribuye a facilitar el proceso de elaboración del duelo en los niños y adolescentes.

Palabras clave: Muerte parental, comunicación, duelo, infancia.
Abstract

Aims: Death is a taboo in our society, and it is common that parents have questions about how to cope with children this issue. The aims of this paper are: 1) to describe the developmental concept of death and the ways of coping in children and adolescents in case of loss 2) to make a literature review of the main recommendations and guidelines to address the communication process and preparation children in this situation.

Method: A systematic literature search has been made in primary and secondary databases (TripDatbase, Psyclnfo, PubMed, Cochrane Library, Psicodoc), by combining different keywords.

Results: Most of the reviewed material comes from England and USA. In our country the majority has an informative format, and we have no evidence of published regulated research. However it is possible to remove from all the material reviewed a number of general guidelines to facilitate communication with children in case of loss of a loved one.

Conclusions: The correct and based on established guidelines communication makes easier the process of grief in children and adolescents.

Keywords: Parental dead, communication, grief, childhood. 


\section{PLANTEAMIENTO DEL PROBLEMA}

La muerte es un tema tabú en la sociedad de hoy en día. La evitación del dolor y de forma más intensa de todo lo relativo a la muerte, lleva a las personas a no abordar este tema con la profundidad necesaria. Así, cuando se produce la pérdida de un ser querido, los adultos se encuentran ante una situación novedosa y sin las estrategias de afrontamiento adecuadas. Con la intención de evitar sufrimiento innecesario y causar el menor impacto posible, en muchas ocasiones se actúa de forma intuitiva o en base a pautas que lejos están de las recomendaciones de las guías y trabajos existentes ${ }^{(1-4)}$.

La comunicación de malas noticias a niños y adolescentes se hace más complicada cuando el fallecido es un familiar muy cercano al menor, como en el caso de los progenitores, y especialmente, cuando el encargado de hacer esta comunicación es el progenitor superviviente. Los trabajos publicados hasta la actualidad han demostrado que una comunicación adecuada cuando tiene lugar la pérdida de un ser querido facilita el proceso de elaboración del duelo y puede evitar su complicación en los niños ${ }^{(5)}$. Cuando el fallecimiento tiene lugar en el ámbito hospitalario, la gestión adecuada de esta comunicación- corresponde no solo a los padres sino también al personal sanitario que ha atendido al fallecido $y$, no es extraño que los profesionales sanitarios tengan dudas acerca de cómo transmitir estas noticias a los menores, dudas que suelen unirse a las de los padres y a las de los demás familiares. En líneas gene- rales, son varios los factores que inciden en la adecuada comunicación de malas noticias: variables relacionadas con el propio paciente o con los familiares, variables relacionadas con el propio centro sanitario etc. ${ }^{(6)}$. Concretamente, las variables relativas a los profesionales sanitarios $y$, en especial, su percepción de competencia, incide directamente en el nivel de estrés laboral percibido ${ }^{(3,6,7)}$. Cabría esperar, por tanto, que la capacitación de los profesionales en este ámbito se asocie por un lado, a una mejora en la calidad asistencial de los servicios sanitarios y también con una disminución en la percepción del burnout ${ }^{(8-9)}$; por lo que el desarrollo de programas estructurados en este ámbito (al igual que ya se ha hecho en el ámbito escolar ${ }^{(37)}$, es un propuesta de futuro.

El objetivo de este trabajo es revisar la bibliografía existente sobre la comunicación a menores cuando ocurre un fallecimiento y en base a ella, definir las pautas de actuación o intervenciones eficaces. Previamente, y con la finalidad de contextualizar dichas pautas en el contexto del desarrollo evolutivo, se hace una revisión general del concepto de muerte en la infancia.

\section{DESARROLLO DEL TEMA}

Se ha llevado a cabo una búsqueda bibliográfica sistemática de las intervenciones eficaces en comunicación de una pérdida a niños y adolescentes en las bases de datos primarias y secundarias (TripDatbase, PubMed, Cochrane Library, Psicodoc y Psyclnfo) empleando la siguiente estrategia de búsqueda y palabras clave:

\footnotetext{
"Bereavement"[Mesh] AND "Professional-Family Relations"[Mesh]) AND

"Bereavement"[Mesh] AND "Adaptation, Psychological"[Mesh])

"Bereavement"[Mesh] AND "Attitude to Death"[Mesh] AND ("infant"[MeSH Terms]

OR "child" [MeSH Terms] OR "adolescent" [MeSH Terms]

("Infant" [MeSH Terms] OR "child" [MeSH Terms] OR "adolescent" [MeSH Terms]

AND "communicating bad news").
} 
En base a la variabilidad de trabajos, y siguiendo los objetivos de este trabajo, se aplicaron como criterios de inclusión los siguientes: 1) La población diana debe estar formada, bien directamente por menores en duelo (o que éste se prevea), o bien por su familia, o por los sanitarios que puedan interactuar con ellos, 2) El objetivo de las intervenciones es aumentar las estrategias de afrontamiento, por parte de los niños o adolescentes, ante el fallecimiento de uno de los progenitores o familiar muy cercano, 3) idioma: inglés o castellano. En cuanto a los criterios de exclusión: aqueIlos trabajos centrados en el duelo parental tras la pérdida de un hijo, dirigidos a profesionales no sanitarios (p.e., profesores), o centrados en el duelo de los niños tras el fallecimiento por suicidio de un familiar (en este supuesto entran en juego otras variables más específicas).

El concepto de muerte en la infancia y adolescencia: respuestas ante la pérdida

En función de su edad, los niños entenderán la muerte de forma diferente. El concepto de muerte cambia con el desarrollo cognitivo, por ello es fundamental adaptar a ellos la información proporcionada. Las diferencias se resumen en $(2,15)$ :

Hasta los 2 años: los niños de estas edades no tienen el concepto de muerte, sin embargo sí perciben la ausencia de la persona fallecida, y son especialmente susceptibles a los cambios que causa la pérdida en su rutina habitual.

De 3 a 6 años: Los niños tienen un pensamiento egocéntrico, literal y mágico. Conciben la muerte como un estado temporal y reversible, en ausencia del concepto de insensibilidad post-morten, irreversabilidad y el de universalidad (16). Por insensibilidad post-morten se entiende que no contemplan la ausencia de cualquier función vital, también piensan que la persona fallecida está simplemente "dormida" (ausencia del concepto de irreversibilidad); mientras que la universalidad es la aceptación de que la muerte afecta a todos los seres vivos ${ }^{(3)}$. Pueden Ilegar a pensar que la muerte o las enfermedades que la causan son algo contagioso. El egocentrismo característico de estas edades puede hacerlos pensar que la muerte ha sido motivada por su culpa. Las reacciones de perplejidad y confusión ante el fallecimiento son las más habituales.

De los 6 a 10 años: Alrededor de los 7 años los menores adquieren el concepto de irreversibilidad e insensibilidad de la muerte, y a los 9 o 10 años el de universalidad. A esta edad empiezan a mostrarse preocupados por el bienestar de familiares cercanos y también por el suyo propio, además de hacerse muchas preguntas relacionadas con la perdida. Pueden aparecer síntomas relacionados de ansiedad por separación $^{(15)}$.

De 10 en adelante: Al comienzo de la adolescencia, el concepto de muerte es similar al de los adultos, son más conscientes de los cambios que puede implicar en su vida y es habitual que se pregunten por su propia muerte y lo que podría implicar ${ }^{(15)}$.

Las fases de elaboración del duelo están determinadas por la edad, así las observadas en niños y adolescentes presentan algunas diferencias con respecto a los adultos $^{(10,17,18)}$. Ordóñez y Lacasta (2004) ${ }^{(19)}$ recogen los hallazgos de las investigaciones actuales y dividen este proceso en tres fases. Una primera de protesta, en la que el niño añora amargamente al progenitor fallecido y llora suplicando que vuelva. A continuación tendría lugar la fase de desesperanza, la expectativa de que el fallecido regrese empieza a disminuir, es frecuente el llanto intermitentemente y un sentimiento de apatía. Por último, se iniciaría la fase de ruptura de vínculo, en la que se empieza a renunciar a parte del vínculo emocional con el fallecido y a mostrar interés por el mundo 
que le rodea. En líneas generales, las tres diferencias entre el duelo adulto y el de los niños en la primera infancia son que los niños utilizan más la negación, mantienen con mayor facilidad la capacidad de disfrutar con situaciones agradables y no pierden la autoestima ${ }^{(19)}$.

Es frecuente que los niños pequeños muestren sintomatología somática (alteraciones del sueño y/o alimentación, enuresis, encopresis y dolores abdominales) o regresión a etapas anteriores del desarroIlo. También son habituales los problemas de conducta, dificultad para concentrarse o atender a tareas escolares, manifestaciones de hiperprotección hacia seres queridos, hiperactividad o apatía, confusión, etc. Entre las reacciones emocionales más usuales se encuentran la irritabilidad, el rechazo, la tendencia al aislamiento, la tristeza, la ansiedad, y el incremento en los miedos en general ${ }^{(15,19-20)}$.

En los adolescentes son más comunes las reacciones psicológicas similares a las manifestadas en los adultos, como por ejemplo la negación o el aislamiento mecanismos de autoprotección que permiten distanciarse temporalmente y disminuir el impacto de la realidad ${ }^{(21)}$.

Aunque en un primer momento estos síntomas pueden llegar a alarmar a los adultos, todos ellos son considerados como reacciones normales de duelo y en general son estrategias temporales que suelen facilitar la elaboración de lo sucedido. La pérdida de un progenitor por sí sola en ausencia de otros factores adicionales no se relaciona con el desarrollo de psicopatología en la edad adulta ${ }^{(22-23)}$. De hecho, a pesar del impacto negativo que implica la pérdida de uno de los progenitores, investigadores como Kennedy y Lloyd-Williams (2009) han encontrado cómo algunos niños dolientes son capaces de desarrollar nuevas habilidades de afrontamiento que promueven su desarrollo personal a partir del proceso de duelo ${ }^{(17)}$.
Los principales factores de riesgo que se han asociado con el desarrollo de duelo patológico a corto o largo plazo son ${ }^{(19,17,24,25-28)}$ :

a. la presencia de antecedentes psicopatológicos en el menor o en el cuidador principal.

b. la simultaneidad de acontecimientos vitales estresantes.

c. una mala relación con el progenitor que ha fallecido.

d. el apego inseguro con el progenitor superviviente.

e. edades entre 10 y 14 años.

f. una mala adaptación del progenitor superviviente.

g. ciertas circunstancias concretas de la muerte (como una muerte violenta o repentina, etc.)

Es importante atender a la frecuencia, duración y repercusión cotidiana de las manifestaciones del menor con la finalidad de detectar signos de complicación del due$\mathrm{lo}^{19,29-30}$. De este modo, el deseo de querer estar únicamente con el progenitor superviviente días después de la pérdida es una reacción habitual, pero la negativa a la separación del padre/madre impidiendo acudir al colegio sería un indicador de alarma.

Las pautas de comunicación y acompañamiento adecuadas están indicadas para toda la población de menores en duelo. Sin embargo, las intervenciones psicológicas específicas sólo se consideran necesarias si los menores presentan factores de riesgo o indicadores de alarma ${ }^{(5,26-27)}$.

La intervención en el duelo en niños y adolescentes: revisión de intervenciones eficaces

En nuestro país se han publicado algunos libros, guías o manuales sobre el abordaje del duelo en los niños y adolescentes $^{(3-4,29,31-33)}$, sin embargo no consta a los autores de este trabajo la publicación 
en nuestro idioma de algún estudio que analice la eficacia de algún programa de comunicación con menores en situaciones de duelo. El aspecto común a la mayoría de las publicaciones tanto españolas como extranjeras es que describen una intervención posterior al fallecimiento, y tratan superficialmente la etapa de preparación y comunicación de malas noticias.

En idioma inglés, si bien se ha publicado numeroso material divulgativo ${ }^{(10-14)}$; son también escasos los estudios sistemáticos sobre la eficacia de las intervenciones ${ }^{(5)}$. Así por ejemplo, Rosner et al. (2010) ${ }^{(5)}$ realiza un metaanalisis de 27 intervenciones en niños en proceso de duelo, y concluyen que éstas son útiles en casos de duelo complicado o cuando los menores presentan algún tipo de sintomatología, sin llegar a producirse una mejoría significativa en los demás casos. Hasta el momento el programa de Sandler et al. (2013) $)^{(11)}$ es el único sobre el que se ha realizado un estudio controlado para comprobar su eficacia. Este programa tiene un formato psicoeducativo enfocado desde modelos cognitivo-conductuales, reforzado con práctica en vivo y tareas para casa. Como contenidos clave, incluye además de la enseñanza de pautas educativas parentales eficaces, pautas de autorregulación emocional y control de la sintomatología ansioso-depresiva en los adultos que conviven con el menor, combinando intervenciones individuales y grupales como en otros programas ${ }^{(34-35)}$. McPherson et al. $(2007)^{(34)}$ plantean un formato grupal de apoyo a los menores deudos, y detectan necesidades tanto generales como específicas, a las que se va a tratar de dar respuesta en las demás intervenciones propuestas.

La investigación apunta a que la intervención dirigida al progenitor superviviente o a los familiares encargados del cuidado de los niños es clave para favorecer el proceso de duelo en los menores ${ }^{(12,28,36)}$. Esta intervención tiene un doble objetivo: promover la adaptación de los adultos y prevenir al mismo tiempo la complicación del duelo en los niños. Podemos encontrar ejemplos de estas iniciativas en los programas grupales, similares a "campamentos", que se llevan a cabo en el Reino Unido o en los Estados Unidos y en las que se suelen incluir tanto a los padres como a los menores ${ }^{(13,37-39)}$. Una de éstas es la de McClatchey et al. (2012)(39), que, si bien no define los principios de lo que sería una intervención eficaz en la comunicación con los menores, destaca como aspectos fundamentales y terapéuticos la cohesión grupal y la expresión emocional. Bachman et al. (2013) ${ }^{(13)}$ emplean también una intervención grupal, en este caso únicamente con los menores (atendiendo a los familiares en sesiones puntuales), y comprueba la eficacia de la intervención un año después, traduciéndose en una mayor adaptación escolar y desarrollo de estrategias de afrontamiento al estrés en aquellos participantes del programa. Sin embargo, en este último trabajo la intervención tiene lugar tras el fallecimiento, por lo que no es posible confirmar que dichas pautas serían también eficaces en la etapa previa. Ambas intervenciones tienen en común el carácter preventivo, ofrecen servicios específicos de atención al duelo y pretenden evitar una derivación innecesaria a los servicios de Salud Mental en aquellos casos en los que no se constata la presencia de psicopatología, pero sí existen factores de riesgo o señales de alarma. Por otra parte, algunos trabajos como el de Kennedy et al. (2008) se centran en la descripción de las necesidades detectadas a partir de la implantación de un programa de duelo en Escocia, sin llegar a definir pautas de actuación ${ }^{(24-25)}$.

Mientras que la evidencia científica sobre la eficacia de las intervenciones con padres o cuidadores es consistente, las intervenciones realizadas con otros miembros de la familia, por ejemplo hermanos, no parecen contribuir de forma significativa a la elaboración del duelo ${ }^{(12)}$. Además 
de los progenitores, también los profesionales sanitarios que se enfrentan diariamente al fallecimiento de pacientes y a la necesidad de responder a las demandas de los familiares, valoran positivamente poder preparar a la familia para hacer frente a esta situación. Al mismo tiempo, esta capacitación de los profesionales disminuye el impacto que esta experiencia puede tener, especialmente en los casos de mayor cercanía con el paciente ${ }^{(7,40)}$. El programa de Keene et al (2010) tiene como objetivo proporcionar apoyo emocional ante estas situaciones, además de pautas de comunicación eficaces con los familiares de la persona fallecida, pero no se ha llevado a cabo una evaluación de su eficacia ${ }^{(7)}$. Todos los trabajos sobre intervenciones descritos hasta el momento se recogen en la tabla 1.

Tabla 1: Análisis de intervenciones

\begin{tabular}{|c|c|c|c|c|}
\hline Autor/Año & Objetivos & Diseño & Muestra & $\begin{array}{c}\text { Aspectos destacados/ } \\
\text { Aspectos no abordados }\end{array}$ \\
\hline $\begin{array}{l}\text { Bachman } \\
(2013)^{(13)}\end{array}$ & $\begin{array}{l}\text { Análisis de la } \\
\text { eficacia de un } \\
\text { programa de } \\
\text { intervención } \\
\text { con niños tras } \\
\text { la pérdida de } \\
\text { un progenitor } \\
\text { en los EE.UU. } \\
\text { (formato cam- } \\
\text { pamento) }\end{array}$ & $\begin{array}{l}\text { Intervención } \\
\text { con el menor } \\
\text { deudo: aprendi- } \\
\text { zaje de habili- } \\
\text { dades de afron- } \\
\text { tamiento } \\
\text { Grupal }\end{array}$ & $\begin{array}{l}68 \text { menores } \\
\text { Edades } 7-17 \\
\text { años } \\
\text { Familiares } \\
\text { (algunas sesio- } \\
\text { nes puntuales) }\end{array}$ & $\begin{array}{l}\text { Eficacia de la intervención } \\
\text { hasta un año después: adap- } \\
\text { tación escolar y desarrollo } \\
\text { de habilidades de afronta- } \\
\text { miento al estrés, entre otras } \\
\text { La intervención es posterior } \\
\text { al fallecimiento del progeni- } \\
\text { tor, no se aborda la prepara- } \\
\text { ción previa. }\end{array}$ \\
\hline Keene $(2010)^{(7)}$ & $\begin{array}{l}\text { Análisis de } \\
\text { un grupo de } \\
\text { debriefing con } \\
\text { menores, reali- } \\
\text { zado por pro- } \\
\text { fesionales de } \\
\text { una unidad de } \\
\text { cáncer infantil } \\
\text { en los EE.UU.: } \\
\text { estudio de } \\
\text { casos }\end{array}$ & $\begin{array}{l}\text { Intervención } \\
\text { con los profe- } \\
\text { sionales: facili- } \\
\text { tar la expresión } \\
\text { emocional y el } \\
\text { aprendizaje de } \\
\text { habilidades de } \\
\text { afrontamiento } \\
\text { para continuar } \\
\text { con la práctica } \\
\text { clínica } \\
\text { Grupal }\end{array}$ & $\begin{array}{l}676 \text { profesio- } \\
\text { nales } \\
\text { de varias dis- } \\
\text { ciplinas }\end{array}$ & $\begin{array}{l}\text { Aspectos eficaces de este } \\
\text { tipo de intervención: soporte } \\
\text { emocional tras el falleci- } \\
\text { miento de los pacientes. } \\
\text { Comunicación con los } \\
\text { familiares como aspecto } \\
\text { fundamental y relacionado } \\
\text { con el impacto y manejo del } \\
\text { fallecimiento. }\end{array}$ \\
\hline $\begin{array}{l}\text { Kennedy et al. } \\
(2008)^{(10)}\end{array}$ & $\begin{array}{l}\text { Análisis del } \\
\text { programa de } \\
\text { apoyo al duelo } \\
\text { de Escocia: es- } \\
\text { tudio de casos }\end{array}$ & $\begin{array}{l}\text { Intervención } \\
\text { con el progeni- } \\
\text { tor deudo y el } \\
\text { menor: diversos } \\
\text { agentes y segui- } \\
\text { miento de las } \\
\text { necesidades } \\
\text { Grupal }\end{array}$ & $\begin{array}{l}6 \text { familias } \\
\text { Edad de los } \\
\text { menores: } \\
\text { inferior a } 18 \\
\text { años }\end{array}$ & $\begin{array}{l}\text { Necesidades detectadas: } \\
\text { apoyo por parte de los } \\
\text { profesionales del servicio, } \\
\text { información sobre la situa- } \\
\text { ción etc. } \\
\text { No se especifican pautas de } \\
\text { actuación ni presta atención } \\
\text { suficiente al papel de los } \\
\text { progenitores. }\end{array}$ \\
\hline
\end{tabular}




\begin{tabular}{|c|c|c|c|c|}
\hline $\begin{array}{l}\text { MacPherson et } \\
\text { al }(2007)^{(34)}\end{array}$ & $\begin{array}{l}\text { Análisis del } \\
\text { programa de } \\
\text { Cuidados Palia- } \\
\text { tivos de Esco- } \\
\text { cia: estudio de } \\
\text { casos }\end{array}$ & $\begin{array}{l}\text { Grupos de } \\
\text { apoyo tras la } \\
\text { pérdida y apo- } \\
\text { yo puntual en } \\
\text { función de las } \\
\text { necesidades del } \\
\text { caso } \\
\text { Grupal }\end{array}$ & $\begin{array}{l}13 \text { niños y } \\
\text { sus corres- } \\
\text { pondientes } \\
\text { familiares y } \\
\text { profesionales } \\
\text { sanitarios. } \\
\text { Edad de los } \\
\text { menores en } \\
\text { el momento } \\
\text { de la pérdida: } \\
\text { 4-16 años }\end{array}$ & $\begin{array}{l}\text { Necesidades detectadas por } \\
\text { los progenitores y los meno- } \\
\text { res: generales (educación so- } \\
\text { bre la muerte) y específicas } \\
\text { (preparación de los menores } \\
\text { previa al fallecimiento, ex- } \\
\text { presión emocional etc.). }\end{array}$ \\
\hline $\begin{array}{l}\text { Mc- } \\
\text { Clatchey(2012) } \\
\text { (39) }\end{array}$ & $\begin{array}{l}\text { Análisis de la } \\
\text { eficacia de un } \\
\text { programa de } \\
\text { intervención } \\
\text { con niños tras } \\
\text { la pérdida de } \\
\text { un progenitor } \\
\text { en los EE.UU. } \\
\text { (formato cam- } \\
\text { pamento): en- } \\
\text { trevistas con los } \\
\text { menores y sus } \\
\text { padres }\end{array}$ & $\begin{array}{l}\text { Intervención } \\
\text { con el menor } \\
\text { deudo y con el } \\
\text { progenitor deu- } \\
\text { do: aprendizaje } \\
\text { de habilidades } \\
\text { de afrontamien- } \\
\text { to en función } \\
\text { de la edad del } \\
\text { menor } \\
\text { Grupal }\end{array}$ & $\begin{array}{l}\text { Dos grupos } \\
\text { en función } \\
\text { de la edad de } \\
\text { los menores } \\
\text { deudos: } 7-11 \\
\text { y } 12-17\end{array}$ & $\begin{array}{l}\text { Dos modalidades de inter- } \\
\text { vención: actividades plan- } \\
\text { teadas como terapéuticas } \\
\text { y actividades tradicionales. } \\
\text { Descripción de los compo- } \\
\text { nentes valorados como efec- } \\
\text { tivos (expresión emocional, } \\
\text { sentimiento de pertenencia). } \\
\text { Descripción superficial de } \\
\text { pautas para progenitores. } \\
\text { Incluye casos de muerte } \\
\text { inesperada o autoinflingida } \\
\text { (asesinato, suicidio). }\end{array}$ \\
\hline $\begin{array}{l}\text { Rosner (2010) } \\
\text { (5) }\end{array}$ & $\begin{array}{l}\text { Metaanálisis } \\
\text { de interven- } \\
\text { ciones con } \\
\text { niños en duelo: } \\
\text { intervenciones } \\
\text { controladas y } \\
\text { no controladas } \\
\text { (anteriores a } \\
\text { 2006) }\end{array}$ & $\begin{array}{l}\text { Intervención } \\
\text { con el progeni- } \\
\text { tor deudo y el } \\
\text { menor: diversos } \\
\text { agentes y segui- } \\
\text { miento de las } \\
\text { necesidades } \\
\text { Grupal/Indivi- } \\
\text { dual }\end{array}$ & $\begin{array}{l}\text { Menores deu- } \\
\text { dos } \\
\text { Progenitores } \\
\text { deudos }\end{array}$ & $\begin{array}{l}\text { Variedad de las interven- } \\
\text { ciones, tamaño de efecto } \\
\text { moderado. Eficacia superior } \\
\text { cuando los menores pre- } \\
\text { sentan sintomatología y no } \\
\text { en los casos de prevención } \\
\text { primaria. } \\
\text { No se dan pautas concretas } \\
\text { de actuación y tampoco se } \\
\text { especifican las pautas para } \\
\text { padres. }\end{array}$ \\
\hline $\begin{array}{l}\text { Sandler (2013) } \\
\text { (11) }\end{array}$ & $\begin{array}{l}\text { Análisis de la } \\
\text { eficacia de un } \\
\text { programa de in- } \\
\text { tervención con } \\
\text { niños en duelo } \\
\text { desarrollado en } \\
\text { los EE.UU. }\end{array}$ & $\begin{array}{l}\text { Intervención } \\
\text { con el proge- } \\
\text { nitor deudo } \\
\text { y el menor: } \\
\text { aprendizaje de } \\
\text { habilidades de } \\
\text { afrontamiento } \\
\text { mediante distin- } \\
\text { tos enfoques y } \\
\text { técnicas durante } \\
12 \text { sesiones } \\
\text { Grupal }\end{array}$ & $\begin{array}{l}156 \text { familias } \\
\text { Menores de } \\
\text { dos grupos de } \\
\text { edad: } 8-12 \\
\text { años y } 12-16 \\
\text { años. }\end{array}$ & $\begin{array}{l}\text { Se mantienen los beneficios } \\
\text { del programa y la aplicación } \\
\text { de las habilidades aprendi- } \\
\text { das a largo plazo: refuerzo } \\
\text { de capacidades parentales } \\
\text { positivas y reducción del } \\
\text { malestar familiar. }\end{array}$ \\
\hline
\end{tabular}


En líneas generales, y de cara a establecer con mayor precisión las consideraciones generales que deberían guiar la comunicación con los menores deudos, algunos de los trabajos revisados coinciden en señalar que ante la muerte las necesidades de los niños y adolescentes son las mismas que las de los adultos ${ }^{24-25,}$ 39), y se hace imprescindible dar respuesta a ellas ${ }^{(3,32)}$. Estas necesidades son:

1. Información: los menores, como cualquier otro miembro de la familia, necesitan que no se les excluya de algo de lo que, aunque doloroso, también forman parte. La exclusión de los menores de este proceso dificulta la elaboración del duelo, independientemente de su edad e incrementa la probabilidad de desarrollar problemas psicológicos en un futuro ${ }^{(41-42)}$. Christ y Christ (2006) destacan además que el proceso de comunicación debe ser (se pueda preveer el fallecimiento), siempre que sea posible, gradual ${ }^{(22)}$. El primer paso para iniciar el proceso de duelo es una explicación sincera y objetiva sobre lo sucedido, así como también explicarles lo que va a ocurrir los días siguientes al faIlecimiento. En el caso de muerte por enfermedad, en especial en la etapa final de la vida, se debe permitir su participación en los cuidados, pudiendo realizar alguna tarea acorde a su edad ${ }^{(19)}$.

2. Normalización y mantenimiento de sus rutinas: las guías coinciden en destacar que lo que más ayuda a los niños frente a la pérdida de un ser querido es recuperar su ritmo cotidiano de actividades, en la escuela y en la relación con sus amigos, teniendo siempre como punto de referencia el ritmo "tolerable" por el menor. El objetivo principal en este momento es asegurar la continuidad en los cuidados, es decir, siempre que sea posible, no se deben realizar cambios importantes en su entorno, como una mudanza, cambio de colegio etc., y procurar que sean las mimas personas las que continúen ocupándose de su atención ${ }^{(43)}$.

3. Ambiente abierto a la expresión de las emociones y en el que reciban apoyo emocional: permitir a los niños expresar la rabia, ira, tristeza o preocupación, como forma de tomar conciencia de sus emociones, explorarlas y ganar control sobre ellas. Dar apoyo, escuchar y validar estas emociones, y transmitir esperanza de recuperación permite una mejor elaboración del duelo. Al mismo tiempo, es necesario que los menores tengan momentos de descanso en los que puedan desconectar del proceso que están viviendo. De hecho, una de las formas de afrontamiento adaptativas más comunes entre los adolescentes es la distracción. La clave está en permitir la ambivalencia del menor entre momentos en los que precisa expresar sus emociones y momentos en los que necesita alejarse emocionalmente ${ }^{(17)}$.

4. Formar parte de los rituales de despedida: permitirles, siempre que lo deseen, despedirse del fallecido. Partiendo de la premisa, apuntada más arriba, de que, a mayor información proporcionada, menor impacto, es necesario explicarles previamente en qué consisten estos actos simbólicos, ya que pueden tener expectativas erróneas sobre lo que se van a encontrar. También es aconsejable que en tales situaciones permanezcan siempre en compañía de algún miembro de la familia que se muestre dispuesto a aclarar sus dudas y a contestar sus preguntas ${ }^{(43)}$.

5. Mantener una conexión simbólica con el ser querido que ha muerto: las investigaciones más referenciadas defienden que las personas en duelo no tienen 
por qué abandonar o renunciar a la relación que mantenían con la persona fallecida, sino que pueden conservar con ésta "una relación simbólica" a lo largo de sus vidas. Se puede ayudar a los niños a redefinir su relación con el ser querido y a preservar sus recuerdos significativos. Esta conexión tiene efectos saludables en el proceso de adaptación tras la pérdida ${ }^{(3)}$.

Las necesidades arriba descritas determinan las pautas generales de comunicación de malas noticias, que se describen a continuación.

¿QUIÉN debe dar la mala noticia?: Si muere uno de los progenitores, Io preferible sería que su cónyuge, siempre que estuviera en condiciones de hacerlo, lo comunicara al niño. Si a los padres les resultara imposible debería hacerlo otra persona emocionalmente cercana al niño y significativa para él, tras explicarle el porqué de la ausencia de los progenitores $^{(1,36)}$.

¿CUÁNDO debe darse la mala noticia?: debe comunicársele al niño inmediatamente o lo antes posible. El niño siempre está alerta, capta lo que está viviendo y de no hacerlo así, podría enterarse por otras personas. Cuando sea posible, debe prepararse al menor ante el fallecimiento que vaya a ocurrir próximamente, anticipándolo con frases como "cada vez está más débil", "Está muy, muy enfermo", "Ya no puede caminar", que ayudarán a los más pequeños a comprender al cambio que va a ocurrir(1,20,36).

¿DÓNDE debe darse la mala noticia?: Preferiblemente, se recomienda hacerlo en un lugar tranquilo y en el que el niño sienta seguro. En el caso de que el fallecimiento se produzca en el hospital, debe buscarse un lugar que reúna estas características $^{(31,20,36)}$.
¿CÓMO debe hacerse?: es importante tener en cuenta estas consideraciones ${ }^{(3,31,36)}$ :

- Mostrar cercanía y mantener un contacto físico apropiado.

- Evitar el uso de eufemismos y de detalles excesivos o explicaciones abstractas. Adaptar la explicación la edad que tenga el menor. Hay que medir mucho las palabras $y$ ser conscientes del modo en que el niño las puede entender. Preguntarle cualquier duda que pueda tener y que puede que no nos exprese.

- Asegurarse de que el menor no se sienta culpable del fallecimiento de la otra persona y combatir el miedo que pueden tener a que otro familiar cercano pueda morir, algo que es bastante frecuente en los niños más pequeños.

- Permitir la expresión emocional propia y mostrar interés por las que pueda expresar el niño. La demostración de que el adulto también está afectado ayuda al niño a normalizar sus reacciones emocionales.

- Describir la rutina de los próximos días, principalmente destacar la continuidad de sus vidas y reducir las dudas que puedan tener al respecto.

El material revisado apunta a que teniendo en cuenta estas necesidades y pautas de actuación a la hora de comunicar a los niños y adolescentes el fallecimiento de un progenitor, facilitaría la elaboración adecuada del duelo. Sin embargo, para comprobar la veracidad de esta hipótesis en el ámbito sanitario, se hace imprescindible desarrollar en el futuro programas estructurados, con componentes y pautas definidas que permitan analizar de forma fiable su eficacia. 


\section{DISCUSIÓN}

En la actualidad, la muerte es todavía un concepto considerado tabú, especialmente cuando se trata de explicar este concepto a los menores. Cuando un adulto se enfrenta a la tarea de tener que informar a un niño o adolescente sobre el fallecimiento de un familiar, y especialmente cuando el fallecido es uno de los progenitores, es común que surjan dudas acerca de cómo abordar esta comunicación, dudas que también aparecen en el personal sanitario que se enfrenta a estas situaciones. El alejar a los menores del sufrimiento y de todo lo relacionado con la muerte, con la intención de facilitar la adaptación a la situación es una creencia errónea y únicamente dificulta la preparación y elaboración posterior del duelo ${ }^{(14)}$; preparación que tiene un impacto positivo no sólo a corto plazo sino también en la etapa adulta ${ }^{(44)}$.

Los trabajos revisados en el presente artículo establecen una serie de directrices claras sobre cómo actuar cuando un niño pierde a un familiar, y en especial sobre la comunicación en el momento posterior al fallecimiento. En este sentido destaca la importancia de establecer una comunicación sincera, adecuar la información a la edad del menor y seguir unas pautas básicas en relación al qué, quién, cuándo, cómo y dónde informar. Las pautas descritas proceden tanto de fuentes de información tradicionales como de páginas web de referencia en otros países ${ }^{(45-47)}$. Esta demanda procede no sólo de los padres u otros familiares, sino también de los profesionales sanitarios encargados de la atención a estos pacientes ${ }^{(48)}$.

Sin embargo, y a pesar de la asunción de que son necesarias unas pautas concretas, son pocas las publicaciones centradas en análisis de la eficacia de las intervenciones, o en describir programas estructurados. Por ello se considera fundamental formar al personal sanitario en el manejo de estas situaciones de la forma más adecuada para el menor y su familia, cuestión que debería ser abordada en futuros trabajos, adecuando también los formatos de los programas a la actualidad.

\section{REFERENCIAS BIBLIOGRÁFICAS}

1. Alelwani SM, Ahmed $Y$ a. Medical training for communication of bad news: A literature review. J Educ Health Promot 2014;3:51; Doi: 10.4103/2277-9531.134737

2. Sociedad Española de Oncología Médica (SEOM). ¿Cómo hablar del cáncer con...? Colección OncoVida. Madrid: Pfizer.

3. Cid Egea L. Explícame qué ha pasado. Guía para ayudar a los adultos a hablar de la muerte y el duelo con los niños. Madrid: Fundación Mario Losantos del Campo, 2011.

4. López de Ayala García C, Galea Martín T, Campos Méndez R. Guía Clínica. Seguimiento del duelo en Cuidados Paliativos. Observatorio Regional de Cuidados Paliativos de Extremadura. Junta de Extremadura, Servicio Extremeño de Salud- FundeSalud,2010.

5. Rosner R, Kruse J, Hagl M. A meta-analysis of interventions for bereaved children and adolescents. Death Studies 2010;34:99136. Doi: 10.1080/07481180903492422.

6. Ptacek JT, McIntosh EG. Physician challenges in communicating bad news. J Behav Med 2009;32:380-7; Doi: 10.1007/ s10865-009-9213-8

7. Keene EA, Hutton N. Hall B, Rushton C. Bereavement debriefing sessions: An intervention to support health care professionals in managing their grief after the death of a patient. Pediatr Nurs 2010;36:185-9.

8. Greenberg LW, Ochsenschlager D, O'Donnell R, Mastruserio J, Cohen GJ. Communicating bad news: A pediatric department's evaluation of a simulated intervention. Pediatrics 1999;103:1210-7. Doi: 10.1542/peds.103.6.1210

9. Vaidya VU, Greenberg LW, Patel KM, Strauss LH, Pollack MM.. Teaching physicians how to break bad news. Arch Pedi- 
atr Adolesc Med 1999;153:419-22; Doi: 10.1001/archpedi.153.4.419.

10. Kennedy C, Mclntyre R, Worth A, Hogg $R$. Supporting children and families facing the death of a parent: part 2. Int J Palliat Nurs 2008;14:230-7. Doi: 10.12968/ ijpn.2008.14.5.29490

11. Sandler I, Wolchik SA, Ayers T S, Tein JY,Luecken L. Family Bereavement Program (FBP) approach to promoting resilience following the death of a parent. Fam Sci 2013;4:1-14, Doi: 10.1080/19424620.20 13.821763.

12. Prchal A, Landolt MA. Psychological interventions with siblings of pediatric cancer patients: A systematic review. Psychooncology 2009;18:1241-51. Doi: 10.1002/ pon.1565.

13. Bachman, B. The development of a sustainable, community-supported children's bereavement camp. Omega (Westport). 2013;67:21-35. Doi: 10.2190/OM.67.1-2.c.

14. Batts J. Death and grief in the family: tips for parents. Helping children at home and school II: Handouts for families and educators. National Association of School Psychologists (NASP) 9:21-29.

15. Biank NM, Werner-Lin A. Growing up with grief: Revisiting the death of a parent over the life course. Omega (Westport) 2011;63:271-90. Doi: 10.2190/OM.63.3.e

16. Piaget J. Seis estudios de Psicología. Madrid: Labor, 1991.

17. Kennedy V. L., Lloyd-Williams M. How children cope when a parent has advanced cancer. Psychooncology 2009;18:886-92. Doi: 10.1002/pon.1455.

18. Kirwin KM, Hamrin V. Decreasing the risk of complicated bereavement and future psychiatric disorders in children. J Child Adolesc Psychiatr Nurs 2005;18:62-78. Doi: 10.1111/j.1744-6171.2005.00002.x.

19. Ordoñez Gallego A, Lacasta Reverte MA. 2004. El duelo en los niños (la pérdida del padre/madre). En: Manual SEOM de Duelo en Oncología. Madrid: Dispublic, SL 2004.p.121-36.
20. National Institute of Mental Health. Talking to children about death. Bethesda: $\mathrm{NIH}$ Clinical Center Patient Education Materials; 2015. [Acceso 7 de octubre de 2015] Disponible en: http://www.cc.nih.gov/ccc/ patient_education/pepubs/childdeath.pdf.

21. Arranz P, Barbero JJ, Barreto P, Bayes R. Intervención emocional en cuidados paliativos: Modeloy protocolos. Barcelona:Ariel, 2003.

22. Christ GH, Christ AE. Current approaches to helping children cope with a parent' s. Cancer J Clin 2006; 56:197-212. Doi: 10.3322/canjclin.56.4.197,

23. Dowdney L. Annotation: Childhood bereavement following parental death. J. Child Psychol Psychiat 2000;41 819-30. Doi: 10.1111/1469-7610.00670.

24. Kennedy C, McIntyre R, Worth A, Hogg R. Supporting children and families facing the death of a parent: Part 2. Int J Palliat Nurs 2008;14:230-7. Doi: 10.12968/ ijpn.2008.14.5.29490

25. Kennedy C, Mclntyre R, Worth A, Hogg R. Supporting children and families facing the death of a parent: Part 1. Int J Palliat Nurs. 2008; 14:162-8. Doi: 10.12968/ ijpn.2008.14.4.29130

26. Schoenfelder EN, Sandler IN, Wolchik S, MacKinnon D. Quality of social relationships and the development of depression in parentally-bereaved youth. J Youth Adolesc 2011; 40:85-96. Doi: 10.1007/s10964009-9503-z.

27. Gray LB, Weller RA, Fristad M, Weller EB. Depression in children and adolescents two months after the death of a parent. J Affect Disord 2011;135: 277-83. Doi: 10.1016/j. jad.2011.08.009

28. Melhem NM, Porta G, Shamseddeen W, Walker M, Payne, M W, Brent DA. The course of grief in children bereaved by sudden parental death. Arch Gen Psychiatry 2011; 68:911-9. Doi: 10.1001/archgenpsychiatry.2011.101.

29. Lacasta MA, Novellas A. Guía de duelo para familiares [libro electrónico]. Madrid: Sociedad Española de Cuidados Paliativos 
(SECPAL), 2014. [último acceso 07 de octubre de 2015]. .Disponible en: en: http:// www.bdv.cat/sites/default/files/common/ Salut/guia_per_a_familiars_en_dol.pdf

30. Lacasta MA, Soler MC. Duelo: prevención y tratamiento del duelo patológico. Cuidados después de la muerte. En: Manual SEOM de Cuidados Continuos. Madrid: Dispublic, SL 2004:713-730, 2004.

31. Quiles Sebastián MJ, Bernabé Castaño M, Esclapés García C, Martín-Aragón Gelabert M, Quiles Marcos Y. Guía Apoyo al duelo [libro electrónico]. Elche: Universidad Miguel Hernández, 2007. Disponible en: http:// donacion.organos.ua.es/submenu3/inf_sanitaria/duelo/LibroApoyoalDueloASV.pdf

32. Kroen WC. Cómo ayudar a los niños a afrontar la pérdida de un ser querido. Manual para adultos. Madrid: Oniro, 2011.

33. Ramos, R. Las estrellas fugaces no conceden deseos. Programa de prevención, evaluación e intervención por duelo en el contexto escolar. Madrid: TEA Ediciones, 2010.

34. MacPherson C, Emeleus M. Children's needs when facing the death of a parent from cancer: part one. Int J Palliat Nurs. 2007;13:478-85.Doi: 10.12968/ ijpn.2007.13.10.27478.

35. MacPherson C, Emeleus M. Children's needs when facing the death of a parent from cancer: part two. Int J Palliat Nurs 2007;13:590-7.Doi: 10.12968/ ijpn.2007.13.12.27887.

36. Werner-Lin A, Biank, NM. Holding parents so they can hold their children: Grief work with surviving spouses to support parentally bereaved children. Omega (Westport) 2012; 66:1-16. Doi: 10.2190/OM.66.1.a.

37. Brewer JD, Sparkes AC. Young people living with parental bereavement: Insights from an ethnographic study of a UK childhood bereavement service. Soc Sci Med 2011;72: 283-90. Doi: 10.1016/j.socscimed.2010.10.032.

38. Lydon A, Hennings J, Woolley BR. Evaluation of a British child bereavement service: the user's perspective. Palliat Support
Care 2010; 8:297-303. Doi: 10.1017/ S147895151000009X.

39. McClatchey IS, Wimmer JS. Healing components of a bereavement camp: Children and adolescents give voice to their experiences. Omega (Westport). 2012; 65:11-32. Doi: 10.2190/OM.65.1.b.

40. Wang YM. Yes, I can learning to cope with death. Pharos Alpha Omega Alpha Honor Med Soc 2013; 15.

41. Macy M. Through the eyes of child: Reflections on my mother's death from cancer. J Pain Palliat Care Pharmacother 2013;27:176-8. Doi: 10.3109/15360288.2013.782938

42. Mendhekar DN, Lohia D. Complicated grief in a two-and-a-half-year-old child. Singapore Med J 2010;51:34-6.

43. American Cancer Society. Coping with the loss of a loved one. Grieving, mourning, and bereavement. New York, 2010.

44. Ellis J, Dowrick C, Lloyd-Williams M. The long-term impact of early parental death: Lessons from a narrative study. J R Soc Med 2013; 106:57-67. Doi: 10.1177/0141076812472623.

45. Winstonwish.org [sede web]. Londres: Wiston Wish; 2015- [actualizada el 7 de octubre de 2015; acceso 7 de octubre de 2015]. Disponible en: http://www.winstonswish.org.uk/

46. Rainbows.org [sede web]. Evanston: Rainbows for all children; 2015- [actualizada el 7 de octubre de 2015; acceso 7 de octubre de 2015]. Disponible en: https://rainbows.org/

47. National Cancer Institute [sede web]. Bethesda: National Cancer Institute; 2015- [actualizada el 7 de octubre de 2015; acceso 7 de octubre de 2015]. Grief, bereavement, and coping with loss; [aproximadamente 7 pantallas]. Disponible en: http://www.cancer. gov/about-cancer/advanced-cancer/caregivers/planning/bereavement-pdq\#section/_62

48. Fearnley R. Death of a parent and the children's experience: Don't ignore the elephant in the room. J Interprofessional Care 2010; 24: 450-9. Doi: 10.3109/13561820903274871shoen. 